\title{
Multiple trait model combining random regressions for daily feed intake with single measured performance traits of growing pigs
}

\author{
Urs SCHNyder $^{\mathrm{a}, *}$, Andreas Hofer ${ }^{\mathrm{a}}$, \\ Florence LABROUE ${ }^{\mathrm{b}}$, Niklaus KÜNZI ${ }^{\mathrm{a}}$ \\ a Institute of Animal Science, Swiss Federal Institute of Technology (ETH), \\ 8092 Zürich, Switzerland \\ b Institut technique du porc, La Motte au Vicomte, \\ BP 3, 35651 Le Rheu cedex, France
}

(Received 14 September 2000; accepted 21 June 2001)

\begin{abstract}
A random regression model for daily feed intake and a conventional multiple trait animal model for the four traits average daily gain on test (ADG), feed conversion ratio (FCR), carcass lean content and meat quality index were combined to analyse data from 1449 castrated male Large White pigs performance tested in two French central testing stations in 1997. Group housed pigs fed ad libitum with electronic feed dispensers were tested from 35 to $100 \mathrm{~kg}$ live body weight. A quadratic polynomial in days on test was used as a regression function for weekly means of daily feed intake and to describe its residual variance. The same fixed (batch) and random (additive genetic, pen and individual permanent environmental) effects were used for regression coefficients of feed intake and single measured traits. Variance components were estimated by means of a Bayesian analysis using Gibbs sampling. Four Gibbs chains were run for 550000 rounds each, from which 50000 rounds were discarded from the burn-in period. Estimates of posterior means of covariance matrices were calculated from the remaining two million samples. Low heritabilities of linear and quadratic regression coefficients and their unfavourable genetic correlations with other performance traits reveal that altering the shape of the feed intake curve by direct or indirect selection is difficult.
\end{abstract}

random regression / variance component / Gibbs sampling / feed intake / pig

\footnotetext{
* Correspondence and reprints

E-mail: urs.schnyder@braunvieh.ch

Present address: Swiss Brown Cattle Breeders' Federation, Chamerstrasse 56, 6300 Zug, Switzerland
} 


\section{INTRODUCTION}

Electronic feeders installed in central testing stations allow for the measurement of individual daily feed intake of performance tested growing pigs. Today's pig selection programs only make use of these data by calculating average daily feed intake as a simple mean of daily feed intake records over the whole testing period. In a previous study we have shown that more information can be retained from these data with a random regression model, using a quadratic polynomial to describe the course of daily feed intake of growing fattening pigs [11]. Genetic eigenfunctions and low heritabilities of linear and quadratic random regression coefficients of daily feed intake indicate that changes of the overall level are easier to achieve than changes of slope or inflexion of feed intake curves. It therefore seems difficult to improve the efficiency of lean growth by selecting for a higher feed intake in the beginning of the fattening period while leaving the feed intake capacity at its present level towards the end [11]. Such an advantage over the use of traditional traits (average daily feed intake, average daily gain and/or the ratio of the two, i.e. feed conversion) for selection of pigs for growth performance would be necessary to justify the use of a random regression model for routine evaluations.

Correlations of random regression coefficients for feed intake with traditional single measured performance traits of growing pigs might help to judge the potential of random regression models for future pig breeding programs. To our knowledge, no attempt has been published to combine a random regression model for a trait with repeated measurements with a conventional multiple trait model for single measured traits in a joint analysis.

The objective of this study was to combine the random regression model previously used for the analysis of daily feed intake data [11] with a multiple trait model for single measured performance traits of growing pigs and to assess possible routes of improvement of the efficiency of lean growth based on estimates of genetic and phenotypic correlations obtained from this joint analysis.

\section{MATERIALS AND METHODS}

\subsection{Data}

1449 castrated Large White pigs were performance tested in two French central testing stations in 1997. Growing pigs were housed in group pens equipped with one electronic feed dispenser each (Acema-48, Acemo, Pontivy, Morbihan, France), where ad libitum daily feed intake was recorded. Groups that were on test during the same period of time on the same testing station formed a batch. There was a total of 155 groups in 13 batches. After one week of adaptation to the automatic feed dispensers, pigs entered the testing phase 
Table I. Number $(n)$ and proportion (\%) of tested animals with records for weekly means of daily feed intake by test week (or corresponding test day).

\begin{tabular}{lcccccccccccccc}
\hline Week & 1 & 2 & 3 & 4 & 5 & 6 & 7 & 8 & 9 & 10 & 11 & 12 & 13 & 14 \\
Day & 4 & 11 & 18 & 25 & 32 & 39 & 46 & 53 & 60 & 67 & 74 & 81 & 88 & 95 \\
\hline$n$ & 1423 & 1444 & 1443 & 1441 & 1442 & 1435 & 1423 & 1407 & 1378 & 1213 & 713 & 225 & 51 & 3 \\
$\%$ & 98.2 & 99.7 & 99.6 & 99.4 & 99.5 & 99.0 & 98.2 & 97.1 & 95.1 & 83.7 & 49.2 & 17.6 & 3.5 & 0.2 \\
\hline
\end{tabular}

with about $35 \mathrm{~kg}$ live body weight and were slaughtered after the end of the test with $100 \mathrm{~kg}$ live body weight on average. Weekly means of feed intake per day were calculated and saved as the record of the middle day of the test week, in order to reduce the amount of data for the evaluations. Whenever records of more than one day per week were missing, all the records of this week were discarded and the weekly mean was set to missing. This resulted in records for days $4,11,18, \ldots, 81,88,95$ (Tab. I). Other traits included in this evaluation were average daily gain and feed conversion ratio calculated for the period between the start and the end of the test, as well as carcass lean content and meat quality index determined after the slaughtering of tested animals.

\subsection{Model}

The following random regression model, which is a quadratic polynomial in days on test $d_{m}$ was fitted to weekly means of daily feed intake records:

$$
\begin{aligned}
y_{i j k m}= & \text { batch }_{0 k}+\text { batch }_{1 k} * d_{m}+\text { batch }_{2 k} * d_{m}^{2} \\
& +a_{0 i}+a_{1 i} * d_{m}+a_{2 i} * d_{m}^{2} \\
& +p_{0 j}+p_{1 j} * d_{m}+p_{2 j} * d_{m}^{2} \\
& +e_{0 i}+e_{1 i} * d_{m}+e_{2 i} * d_{m}^{2} \\
& +\varepsilon_{i j k m}
\end{aligned}
$$

where batch $_{n k}$ are fixed regressions for the period and station of test; $a_{n i}$ are random regressions for animal additive genetic effects; $p_{n j}$ and $e_{n i}$ are random regressions for permanent environmental effects of pen and the tested individual, respectively; $\varepsilon_{i j k m}$ is a random residual error which accounts for daily deviations of feed intake from the expected trajectory of animal $i$ on day $d_{m}$. What is called "permanent environmental effect of the tested individual", is a residual for regression coefficients. This random regression model corresponds to the one used in a previous analysis of daily feed intake records of performance tested growing pigs [11]. Fixed regression coefficients due to the gender of the animals as well as random regression coefficients due to litter permanent environmental effects were dropped from the model, since only castrated males were tested, which usually had no litter mates in the test. 
Daily deviations from the estimated feed intake curve of an animal (residuals $\left.\varepsilon_{i j k m}\right)$ were assumed to be independent of each other. All the animals were assumed to have the same residual variance for feed intake on a given day on test $d_{m}$, which was modelled as follows:

$$
\sigma_{\varepsilon_{m}}^{2}=\exp \left(\gamma_{0}+\gamma_{1} * d_{m}+\gamma_{2} * d_{m}^{2}\right)
$$

In a previous analysis, this model for the residual variance proved to be better than a constant residual variance over the whole testing period [11]. Changes in the magnitude of the residual variance are mainly due to scale effects, since daily feed intake of pigs increases with stomach and gut size during the growing period.

The model for single measured performance traits average daily gain, feed conversion ratio, carcass lean content and meat quality index contains the same fixed and random effects as for regression coefficients for weekly means of daily feed intake. Additionally, the live weight at the end of the test (before slaughtering) was included as a covariable for average daily gain and feed conversion ratio:

$$
y_{n i j k}=\beta_{n} * \text { weight }_{i}+\text { batch }_{n k}+a_{n i}+p_{n j}+e_{n i}+\varepsilon_{n i j k}
$$

where $y_{n i j k}$ is the record for trait $n$ of animal $i$ in pen $j$ and batch $k . \beta_{n}$ is the regression of trait $n$ on the covariable "weight at the end of the test". For the combination of the two models, additive genetic $\left(a_{n i}\right)$ and permanent environmental effects of the pen $\left(p_{n j}\right)$ of single measured traits $(n)$ are assumed to be correlated with the corresponding effects for random regression coefficients for daily feed intake. Since residuals for regression coefficients are fitted explicitly as individual permanent environmental effects in the random regression model for daily feed intake, such individual permanent environmental effects $\left(e_{n i}\right)$ were also fitted for single measured traits. Individual permanent environmental effects are assumed to be correlated among single measured traits and regression coefficients for feed intake. The residuals $\varepsilon_{n i j k}$ of single measured traits correspond to the residuals $\varepsilon_{i j k m}$ in equation (1), which account for deviations of daily feed intake from the expected trajectory. Residuals $\varepsilon_{n i j k}$ of single measured traits are assumed to be normally distributed and independent of each other as well as from residuals of daily feed intake. The two residual terms in model (3) for single measured traits $\left(e_{n i}\right.$ and $\left.\varepsilon_{n i j k}\right)$ were included to reach compatibility with the random regression model (1) for daily feed intake. Explicitly fitting individual permanent environmental effects $e_{n i}$ in a random regression model is necessary for a proper definition of heritabilities of regression coefficients, since they play the role of residuals for these artificial traits [11]. If one desires to allow for correlations between these explicitly fitted residuals of regression coefficients and residuals of single measured traits in a joint analysis, the only 
possibility is to fit individual permanent environmental effects explicitly for single measured traits as well.

Normal distribution of feed intake data and single measured performance traits is assumed:

$$
\mathbf{y} \mid \mathbf{b}, \mathbf{a}, \mathbf{p}, \mathbf{e}, \sigma_{\varepsilon_{n m}}^{2} \sim N\left\{\mathbf{X b}+\mathbf{Z a}+\mathbf{V} \mathbf{p}+\mathbf{W e}, \mathbf{I} \sigma_{\varepsilon_{n m}}^{2}\right\}
$$

$\mathbf{y}$ is a vector containing data for all traits; $\mathbf{b}$ is a vector containing fixed effects for batch and regressions $\beta_{n}$ on the covariable weight at the end of the test; $\mathbf{a}$ is the vector of additive genetic effects; $\mathbf{p}$ and $\mathbf{e}$ are vectors containing permanent environmental effects; $\mathbf{X}, \mathbf{Z}, \mathbf{V}$ and $\mathbf{W}$ are incidence matrices; $\mathbf{I}$ is the identity matrix and $\sigma_{\varepsilon_{n m}}^{2}$ is the residual variance around feed intake curves for day on test $d_{m}$, or the variance of uncorrelated residuals for single measured traits, respectively.

The following assumptions were used for the distributions of fixed and random effects:

$$
\begin{aligned}
\mathbf{b} & \sim \text { constant } \\
\mathbf{a} \mid \mathbf{A}, \mathbf{G}_{\mathbf{0}} & \sim N\left\{\mathbf{0},\left(\mathbf{A} \otimes \mathbf{G}_{\mathbf{0}}\right)\right\} \\
\mathbf{p} \mid \mathbf{P}_{\mathbf{0}} & \sim N\left\{\mathbf{0},\left(\mathbf{I} \otimes \mathbf{P}_{\mathbf{0}}\right)\right\} \\
\mathbf{e} \mid \mathbf{E}_{\mathbf{0}} & \sim N\left\{\mathbf{0},\left(\mathbf{I} \otimes \mathbf{E}_{\mathbf{0}}\right)\right\}
\end{aligned}
$$

where $\mathbf{A}$ is the numerator relationship matrix, $\mathbf{G}_{\mathbf{0}}$ is the (co)variance matrix of random additive genetic effects and $\mathbf{P}_{\mathbf{0}}$ and $\mathbf{E}_{\mathbf{0}}$ are (co)variance matrices for random permanent environmental effects. All these (co)variance matrices are of dimension $7 \times 7$ (three regression coefficients plus four single measured traits).

Informative priors with low numbers of degrees of freedom were used for the variance components. For the $7 \times 7$ (co)variance matrices $\mathbf{G}_{\mathbf{0}}, \mathbf{P}_{\mathbf{0}}$ and $\mathbf{E}_{\mathbf{0}}$, inverse Wishart distributions with nine degrees of freedom were used. Scale parameters for inverse Wishart prior distributions (Tabs. II and III) were chosen such that resulting expected values of covariance matrices corresponded to our expectation. Expected values for (co)variances of feed intake regression coefficients were taken from our results of an earlier study [11], while genetic and permanent environmental (co)variances for single measured performance traits were derived from Labroue et al. [7]. Their results for average daily feed intake were used for genetic correlations between single measured traits and the intercept of feed intake curves. Priors for genetic covariances of single measured performance traits with linear and quadratic regression coefficients of daily feed intake were set to zero (Tab. II), since no prior information about their true value was available. For simplicity, prior values of all permanent environmental covariances of single measured traits were also set to zero (Tab. III). Total permanent environmental (co)variance (Tab. III) was divided 
Table II. Lower diagonal elements of the symmetric scale matrix $\mathbf{S}_{\mathbf{G}}$ for the inverse Wishart prior distribution of the additive genetic covariance matrix $\left(\mathbf{G}_{\mathbf{0}}\right)$ between the intercept, linear and quadratic regression coefficients for daily feed intake and single measured performance traits: average daily gain $(A D G)$, feed conversion ratio $(F C R)$, carcass lean content $(C L C)$ and meat quality index $(M Q I)$.

\begin{tabular}{lrrrrrrr}
\hline Trait & \multicolumn{1}{c}{ Intercept } & Linear & Quadratic & $A D G$ & $F C R$ & CLC & MQI \\
\hline Intercept & $2.23 \mathrm{e}-2$ & & & & & & \\
Linear & $-3.60 \mathrm{e}-4$ & $1.40 \mathrm{e}-5$ & & & symmetric & & \\
Quadratic & $2.90 \mathrm{e}-6$ & $-7.00 \mathrm{e}-8$ & $1.90 \mathrm{e}-9$ & & & & \\
$A D G$ & 4.90 & 0.0 & 0.0 & 3386.0 & & & \\
$F C R$ & $1.90 \mathrm{e}-3$ & 0.0 & 0.0 & -2.186 & 0.0080 & & \\
$C L C$ & $-1.562 \mathrm{e}-1$ & 0.0 & 0.0 & 0.0 & -0.0990 & 7.620 & \\
$M Q I$ & $1.486 \mathrm{e}-2$ & 0.0 & 0.0 & 0.0 & 0.0141 & -0.1451 & 1.105 \\
\hline
\end{tabular}

Table III. Lower diagonal elements of the symmetric scale matrix $\mathbf{S}_{\mathbf{P E}}$ for the inverse Wishart prior distribution of the total permanent environmental covariance matrix (sum of $\mathbf{P}_{\mathbf{0}}$ and $\mathbf{E}_{\mathbf{0}}$ ) between the intercept, linear and quadratic regression coefficients for daily feed intake and single measured performance traits: average daily gain $(A D G)$, feed conversion ratio $(F C R)$, carcass lean content $(C L C)$ and meat quality index $(M Q I)$.

\begin{tabular}{lrrrrrrr}
\hline Trait & \multicolumn{1}{c}{ Intercept } & \multicolumn{1}{c}{ Linear } & Quadratic & $A D G$ & $F C R$ & $C L C$ & $M Q I$ \\
\hline Intercept & $3.06 \mathrm{e}-2$ & & & & & & \\
Linear & $-1.14 \mathrm{e}-3$ & $1.96 \mathrm{e}-4$ & & & symmetric & \\
Quadratic & $1.11 \mathrm{e}-5$ & $-2.62 \mathrm{e}-6$ & $3.97 \mathrm{e}-8$ & & & & \\
$A D G$ & 0.0 & 0.0 & 0.0 & 5079.0 & & & \\
$F C R$ & 0.0 & 0.0 & 0.0 & 0.0 & 0.032 & & \\
$C L C$ & 0.0 & 0.0 & 0.0 & 0.0 & 0.0 & 3.267 & \\
$M Q I$ & 0.0 & 0.0 & 0.0 & 0.0 & 0.0 & 0.0 & 4.418 \\
\hline
\end{tabular}

into its components pen $\left(\mathbf{P}_{\mathbf{0}}\right)$ and individual $\left(\mathbf{E}_{\mathbf{0}}\right)$ permanent environmental (co)variance with a ratio of 1 to 9 . Priors for parameters $\gamma_{0}, \gamma_{1}$ and $\gamma_{2}$, that describe the course of the residual variance $\sigma_{\varepsilon_{m}}^{2}$ for weekly means of daily feed intake, were assumed independent of each other and normally distributed with standard deviations of $1.5\left(\gamma_{0}\right), 0.1\left(\gamma_{1}\right)$ and $0.01\left(\gamma_{2}\right)$. These standard deviations represent a relatively wide range of values, that parameters $\gamma_{0}, \gamma_{1}$ and $\gamma_{2}$ might reasonably take. The same values were used in an earlier study [11], where they were chosen to express the low level of knowledge about distributions of these parameters. As the Metropolis-Hastings algorithm performed well with these values, they were not changed for the present study.

Unlike residuals for daily feed intake in a random regression model, uncorrelated residuals for single measured traits cannot be distinguished from indi- 
vidual permanent environmental effects. To avoid difficulties of distribution of variance between the two environmental effects of single measured traits, the residual variance $\sigma_{\varepsilon_{n}}^{2}$ was not estimated, but fixed to a value 10000 times smaller than the expected phenotypic variance of the trait. This computational trick forced the residual variance of single measured traits to be attributed to the individual permanent environmental (co)variance matrix $\mathbf{E}_{\mathbf{0}}$. This is illustrated below for two traits with repeated and single measurements, respectively. Suppose the true permanent environmental and residual (co)variance structures for these two traits are given by:

$$
\mathbf{E}_{\mathbf{0}}=\left[\begin{array}{cc}
\sigma_{e_{1}}^{2} & \sigma_{e_{1,2}} \\
\sigma_{e_{1,2}} & \sigma_{e_{2}}^{2}
\end{array}\right] ; \mathbf{R}=\mathbf{I} \sigma_{\varepsilon_{n}}^{2}=\left[\begin{array}{cc}
\sigma_{\varepsilon_{1}}^{2} & 0 \\
0 & \sigma_{\varepsilon_{2}}^{2}
\end{array}\right] .
$$

If the residual variance can be estimated for the trait with repeated measurements (trait 1) and is fixed to a small value $s^{2}$ (smaller than the true value) for the single measured trait (trait 2), the above components will be estimated as:

$$
\mathbf{E}_{\mathbf{0}}=\left[\begin{array}{cc}
\sigma_{e_{1}}^{2} & \sigma_{e_{1,2}} \\
\sigma_{e_{1,2}}\left(\sigma_{e_{2}}^{2}+\sigma_{\varepsilon_{2}}^{2}-s^{2}\right)
\end{array}\right] ; \mathbf{R}=\left[\begin{array}{cc}
\sigma_{\varepsilon_{1}}^{2} & 0 \\
0 & s^{2}
\end{array}\right] .
$$

The major part of residuals of the single measured trait will thus be included in explicitly fitted permanent environmental effects, if the mixed model equations are built with these (co)variance components. As long as the value chosen for $s^{2}$ is smaller than the (unknown) true residual variance of the single measured traits, estimates of covariances in (7) will certainly be unbiased. As long as the permanent environmental correlation calculated from $\mathbf{E}_{\mathbf{0}}$ in equation (7) does not reach the limits of the parameter space, even higher values than the true residual variance can be chosen for $s^{2}$. The following conditions must always hold:

$$
\begin{gathered}
-1 \leqslant \frac{\sigma_{e_{1,2}}}{\sqrt{\sigma_{e_{1}}^{2}\left(\sigma_{e_{2}}^{2}+\sigma_{\varepsilon_{2}}^{2}-s^{2}\right)}} \leqslant 1 \\
\Rightarrow 0 \leqslant \frac{\left(\sigma_{e_{1,2}}\right)^{2}}{\sigma_{e_{1}}^{2}\left(\sigma_{e_{2}}^{2}+\sigma_{\varepsilon_{2}}^{2}-s^{2}\right)} \leqslant 1 \\
\Rightarrow 0<s^{2} \leqslant \sigma_{e_{2}}^{2}+\sigma_{\varepsilon_{2}}^{2}-\frac{\left(\sigma_{e_{1,2}}\right)^{2}}{\sigma_{e_{1}}^{2}} .
\end{gathered}
$$

The value zero is not allowed for $s^{2}$ because $\mathbf{R}$ in (7) has to be positive definite.

\subsection{Variance component estimation}

For the estimation of (co)variance components, our own programs were used applying Bayesian methodology using Gibbs sampling. The joint posterior 
distribution of the parameters given the data is the product of the likelihood and the prior distributions of all parameters. From there, marginal distributions are derived easily, as they only have to be known up to proportionality. This results in normal distributions for solutions of covariables, fixed and random effects and in inverse Wishart distributions for the (co)variance matrices for additive genetic and permanent environmental effects. The parameters $\gamma_{0}, \gamma_{1}$ and $\gamma_{2}$, that describe the course of the residual variance $\sigma_{\varepsilon_{m}}^{2}$, had to be sampled via a Metropolis-Hastings algorithm, as their distribution is not a standard one. A detailed description of the procedure used can be found in Schnyder et al. [11]. Mixed model equations (MME) were processed block-wise by means of Cholesky decomposition and backsubstitution when generating new solutions in the Gibbs sampler. The data was analysed including (model 1) and excluding (model 2) "weight at the end of the test" as a covariable for the single measured traits average daily gain and feed conversion ratio, to investigate the influence of this covariable on heritability estimates. For both models, four Gibbs chains were run, with 550000 samples each.

\subsection{Post-Gibbs analysis}

Burn-in was determined for all (co)variances by the method of Raftery and Lewis [10], using their Fortran program "gibbsit". Additionally, line plots of samples of (co)variance components from every 100th round of Gibbs sampling were used to check convergence of parameters to their stationary distributions. For graphical analysis of Gibbs chains, the statistical software package S-Plus [8] was used. Samples from the burn-in period of each chain were discarded, and posterior means calculated from the remaining samples served as estimates of (co)variance components.

Heritabilities, and genetic and phenotypic correlations were calculated from samples of (co)variance components. For regression coefficients for feed intake, the phenotypic covariance matrix is defined as the sum of additive genetic $\left(\mathbf{G}_{\mathbf{0}}\right)$ and permanent environmental $\left(\mathbf{P}_{\mathbf{0}}, \mathbf{E}_{\mathbf{0}}\right)$ covariance matrices [11]. For single measured traits, the residual variance is also included, i.e. the fixed value $s^{2}$ from equation (7) is added to the sum of estimated additive genetic and permanent environmental variances. For heritabilities, genetic and phenotypic correlations, effective sample size [12] and standard errors of posterior means (Monte Carlo errors) were estimated using estimates of Monte Carlo variance obtained by the method of the initial monotone sequence estimator [3]. This estimator was preferred by Geyer [3] over the initial positive sequence estimator, because it makes large reductions in the worst overestimates while doing little to underestimates. Each Gibbs chain was processed separately, using samples after burn-in only. Estimates of effective sample size were summed over the four Gibbs chains. The variance of an arithmetic mean of $n$ independent values is equal to the original variance of these values divided 
by $n$ (see e.g. [13]). Therefore, estimates of standard errors of overall estimates of posterior means of (co)variance components, are obtained by averaging estimates of standard errors of posterior means of the four individual chains, and dividing this average by two.

(Co)variances between daily feed intake records and single measured performance traits were calculated from posterior means of (co)variance matrices of random regression coefficients for feed intake and single measured performance traits as shown in equation (9) below for additive genetic (co)variances:

$$
\mathbf{C}_{\mathbf{G}}=\boldsymbol{\Phi} \mathbf{G}_{\mathbf{0}} \boldsymbol{\Phi}^{\prime} ; \quad \boldsymbol{\Phi}=\left[\begin{array}{cc}
\boldsymbol{\Phi}_{\mathbf{m}} & \mathbf{0} \\
\mathbf{0} & \mathbf{I}_{\mathbf{n}}
\end{array}\right] ; \quad \boldsymbol{\Phi}_{\mathbf{m}}=\left[\begin{array}{ccc}
1 & d_{1} & d_{1}^{2} \\
\vdots & \vdots & \vdots \\
1 & d_{m} & d_{m}^{2}
\end{array}\right]
$$

where $\mathbf{C}_{\mathbf{G}}$ is a matrix containing genetic (co)variances between daily measurements of feed intake and single measured performance traits of dimension $(m+n)$ rows by $(m+n)$ columns, where $m$ is the number of days (weeks) with measurements of feed intake and $n$ is the number of single measured traits; $\mathbf{G}_{\mathbf{0}}$ is the genetic (co)variance matrix between the 3 random regression coefficients for daily feed intake and the $n$ single measured traits; $\boldsymbol{\Phi}$ is a matrix of $(m+n)$ rows by $(3+n)$ columns consisting of ( $m$ by 3$)$ matrix $\boldsymbol{\Phi}_{\mathbf{m}}$ containing covariables for quadratic polynomials $\left(1\right.$, day, day $\left.{ }^{2}\right)$ for each day with feed intake records in the upper left corner and the $(n$ by $n)$ identity matrix $\mathbf{I}_{\mathbf{n}}$ in the lower right corner, with zeros everywhere else. If $\mathbf{G}_{\mathbf{0}}$ is split into its submatrices corresponding to (co)variances of regression coefficients for feed intake $\left(\mathbf{G}_{\mathbf{1}, \mathbf{1}}\right)$, (co)variances of single measured traits $\left(\mathbf{G}_{\mathbf{2}, \mathbf{2}}\right)$ and covariances between regression coefficients and single measured traits $\left(\mathbf{G}_{\mathbf{1}, \mathbf{2}}\right), \mathbf{C}_{\mathbf{G}}$ can be written as follows:

$$
\mathbf{C}_{\mathbf{G}}=\left[\begin{array}{cc}
\Phi_{\mathrm{m}} \mathbf{G}_{1,1} \Phi^{\prime}{ }_{\mathrm{m}} & \Phi_{\mathrm{m}} \mathbf{G}_{1,2} \\
\mathbf{G}_{\mathbf{2}, \mathbf{1}} \Phi_{\mathrm{m}}^{\prime} & \mathbf{G}_{2,2}
\end{array}\right]
$$

Residual variances around feed intake curves were calculated for the same $m$ days with measurements of feed intake according to equation (2), using posterior means of parameters $\gamma_{0}, \gamma_{1}$ and $\gamma_{2}$. The sum of calculated additive genetic $\left(\mathbf{C}_{\mathbf{G}}\right)$ and permanent environmental $\left(\mathbf{C}_{\mathbf{P}}\right.$ and $\left.\mathbf{C}_{\mathbf{E}}\right)$ (co)variance matrices, with residual variances around feed intake curves $\left(\sigma_{\varepsilon_{m}}^{2}\right)$ added to variances of daily feed intake and fixed residual variances $\left(\sigma_{\varepsilon_{n}}^{2}\right)$ added to variances of single measured traits, yields the phenotypic (co)variance matrix $\mathbf{C}$ between weekly means of daily feed intake and single measured performance traits:

$$
\mathbf{C}=\mathbf{C}_{\mathbf{G}}+\mathbf{C}_{\mathbf{P}}+\mathbf{C}_{\mathbf{E}}+\left[\begin{array}{cc}
\mathbf{I}_{\mathbf{m}} \sigma_{\varepsilon_{m}}^{2} & \mathbf{0} \\
\mathbf{0} & \mathbf{I}_{\mathbf{n}} \sigma_{\varepsilon_{n}}^{2}
\end{array}\right]
$$

From additive genetic and phenotypic (co)variance matrices, heritabilities, genetic and phenotypic correlations were calculated. Course of variances and 
heritability for weekly means of daily feed intake, genetic and phenotypic correlations between weekly means of daily feed intake, as well as their correlations to single measured traits, were plotted for the whole testing period.

\section{RESULTS AND DISCUSSION}

\subsection{Behaviour of the Gibbs sampler}

Burn-in periods estimated with the fortran program "gibbsit" by Raftery and Lewis [10] differed substantially between parameters, chains and specified quantiles of interest. The highest estimates were found for the estimation of 50\%-quantiles of genetic variances of the single measured traits: carcass lean content and meat quality index. Based on these estimates and after graphically checking whether Gibbs chains had converged to a stationary distribution, 50000 rounds of burn-in were chosen for all parameters of all chains.

Sums of estimates of effective sample size per Gibbs chain (Tabs. IV and V) were very low compared to the 500000 rounds of Gibbs sampling run after burn-in for each chain (2000000 samples total). The estimate of effective sample size for the phenotypic correlation between average daily gain (ADG) and feed conversion ratio (FCR) in model 1 with the covariable "weight at the end of the test" for ADG and FCR (Tab. IV), which was much lower than for model 2 without a covariable for ADG and FCR was especially surprising (Tab. V). A possible reason for this low estimate of effective sample size for the phenotypic correlation between ADG and FCR may be found in the special interrelations between these traits. FCR is average daily feed intake divided by ADG and ADG is the weight at the end of the test minus weight at the start, divided by the number of days on test, i.e. both traits are ratios and the covariable specified for both traits is involved too.

The reason for the generally slow mixing of Gibbs chains can be found in fixing the residual variance to a small value and explicitly fitting individual permanent environmental effects for single measured traits. With such a model, traits are fitted almost perfectly by the specified effects, which reduces the freedom of the sampler to change a single effect. This was confirmed by the convergence of a Gauss-Seidel algorithm with a simulated data set. The calculations involved in Gibbs sampling of fixed and random effects are almost identical to the calculations used in the Gauss-Seidel algorithm for solving the mixed model equations. Convergence of a Gauss-Seidel algorithm and mixing of the Gibbs sampler for a given model are therefore closely related. A data set was generated according to a model similar to our model for single measured traits (3), assigning relative values of 70 to the individual permanent environmental variance and 30 to the residual variance. The mixed model equations for this data were then set up using values 99.9 and 0.1 for individual 
Table IV. Estimates of effective sample size (sum over four Gibbs chains) for heritabilities (bold), genetic (above diagonal) and phenotypic (below diagonal) correlations of intercept, linear and quadratic regression coefficients for daily feed intake, and for single measured performance traits: average daily gain $(A D G)$, feed conversion ratio $(F C R)$, carcass lean content $(C L C)$ and meat quality index $(M Q I)$. Model 1 with covariable "weight at the end of the test" for $A D G$ and $F C R$.

\begin{tabular}{lrrrrrrr}
\hline Trait & Intercept & Linear & Quadratic & $A D G$ & $F C R$ & $C L C$ & $M Q I$ \\
\hline Intercept & $\mathbf{8 8}$ & 78 & 95 & 104 & 30 & 60 & 63 \\
Linear & 2280 & $\mathbf{7 0}$ & 84 & 71 & 49 & 62 & 75 \\
Quadratic & 1520 & 2532 & $\mathbf{5 9}$ & 67 & 33 & 50 & 45 \\
$A D G$ & 513 & 605 & 433 & $\mathbf{2 9}$ & 20 & 34 & 47 \\
$F C R$ & 1273 & 1388 & 2376 & 15 & $\mathbf{2 1}$ & 34 & 42 \\
$C L C$ & 814 & 355 & 280 & 246 & 179 & $\mathbf{2 9}$ & 24 \\
$M Q I$ & 1645 & 1140 & 994 & 405 & 717 & 147 & $\mathbf{2 1}$ \\
\hline
\end{tabular}

Table V. Estimates of effective sample size (sum over four Gibbs chains) for heritabilities (bold), genetic (above diagonal) and phenotypic (below diagonal) correlations of intercept, linear and quadratic regression coefficients for daily feed intake, and for single measured performance traits: average daily gain $(A D G)$, feed conversion ratio $(F C R)$, carcass lean content $(C L C)$ and meat quality index $(M Q I)$. Model 2 without a covariable for $A D G$ and $F C R$.

\begin{tabular}{lrrrrrrr}
\hline Trait & Intercept & Linear & Quadratic & $A D G$ & $F C R$ & $C L C$ & $M Q I$ \\
\hline Intercept & $\mathbf{3 9}$ & 22 & 34 & 79 & 35 & 39 & 40 \\
Linear & 2602 & $\mathbf{7 6}$ & 46 & 20 & 39 & 68 & 75 \\
Quadratic & 3142 & 2418 & $\mathbf{3 0}$ & 56 & 26 & 44 & 63 \\
$A D G$ & 772 & 835 & 686 & $\mathbf{2 8}$ & 22 & 39 & 17 \\
$F C R$ & 1277 & 1074 & 1033 & 197 & $\mathbf{2 1}$ & 33 & 26 \\
$C L C$ & 629 & 471 & 352 & 106 & 209 & $\mathbf{2 9}$ & 33 \\
$M Q I$ & 598 & 978 & 735 & 213 & 415 & 138 & $\mathbf{2 6}$ \\
\hline
\end{tabular}

p.e. and residual variances, respectively, and solved using the Gauss-Seidel algorithm. The solutions were the same as for the mixed model equations set up using the true values for variance components, but it took many more rounds to reach the convergence criteria. On the contrary, convergence was much faster if individual permanent environmental effects were not fitted explicitly, but only taken into account by assigning a value of 100 to the residual variance, i.e. the sum of the true individual p.e. and residual variances. Such a parameterisation was used by Meyer et al. [9] for a joint analysis of two traits with single and repeated measurements, respectively. This would certainly also improve the mixing of the Gibbs sampler for our single measured traits, but does not 
Table VI. Averages $(\mu)$ and standard deviations (s.d.) over all tested animals for intercept, linear and quadratic regression coefficients of daily feed intake (coefficients fitted to records of each animal separately) and for single measured performance traits, average daily gain $(A D G)$, feed conversion ratio $(F C R)$, carcass lean content $(C L C)$ and meat quality index $(M Q I)$, together with estimates of posterior mean of phenotypic standard deviations from the two models with $\left(\sigma_{\mathrm{ph}} 1\right)$ and without $\left(\sigma_{\mathrm{ph}} 2\right)$ covariable "weight at the end of the test" for $A D G$ and $F C R$.

\begin{tabular}{|c|c|c|c|c|c|c|c|}
\hline $\begin{array}{l}\text { Trait } \\
\text { Unit }\end{array}$ & $\begin{array}{c}\text { Intercept } \\
\mathrm{kg}\end{array}$ & $\begin{array}{l}\text { Linear } \\
\mathrm{kg} / \text { day }\end{array}$ & $\begin{array}{l}\text { Quadratic } \\
\text { kg/day }{ }^{2}\end{array}$ & $\begin{array}{c}A D G \\
\mathrm{~g}\end{array}$ & $\begin{array}{r}F C R \\
\mathrm{~kg} / \mathrm{kg}\end{array}$ & $\begin{array}{l}C L C \\
\%\end{array}$ & $\begin{array}{l}M Q I \\
-\end{array}$ \\
\hline$\mu$ & 1.344 & $3.60 e-02$ & $-1.47 \mathrm{e}-04$ & 851.74 & 2.918 & 56.67 & 10.734 \\
\hline s.d. & 0.364 & $2.32 \mathrm{e}-02$ & $3.33 \mathrm{e}-04$ & 87.85 & 0.234 & 3.37 & 2.565 \\
\hline$\sigma_{\mathrm{ph}} 1$ & 0.250 & $1.48 \mathrm{e}-02$ & $1.94 \mathrm{e}-04$ & 67.82 & 0.274 & 2.64 & 2.449 \\
\hline$\sigma_{\mathrm{ph}} 2$ & 0.256 & $1.49 \mathrm{e}-02$ & $1.96 \mathrm{e}-04$ & 83.56 & 0.217 & 2.64 & 2.447 \\
\hline
\end{tabular}

allow for residual correlations between random regression coefficients and single measured performance traits, as explicitly fitting individual permanent environmental effects for regression coefficients is necessary for a proper definition of heritability for these artificial traits [11]. Fixing residual variances to higher values than the ones used in this study would improve mixing of the Gibbs sampler. One needs to make sure though that estimates of individual permanent environmental covariances are not affected by the choice of fixed residual variances (see equation (8)).

The following strategy is recommended for the analysis of a random regression model combined with single measured traits:

1) run a short Gibbs chain with the residual variance of single measured traits fixed to a small value ( $s^{2} \sim 1-10 \%$ of phenotypic variance) to get an indication of the distribution of variance among effects;

2) if necessary, adjust $s^{2}$ based on individual permanent environmental correlations (equation (8), new $s^{2}$ higher if correlation close to zero and lower if close to $(-1)$ or 1$)$;

3) then run the Gibbs sampler for as many rounds as needed for the desired accuracy of estimates.

Table VI compares model estimates of phenotypic standard deviations (Gibbs posterior means) with a simple estimate of standard deviation from the raw data (not corrected for fixed effects). For regression coefficients of daily feed intake raw data estimates were obtained by first fitting a quadratic polynomial to feed intake records of each animal separately and then treating the resulting regression coefficients like single measured traits. Mean values (Tab. VI) of intercept and linear regression coefficients for daily feed intake are positive, while it is negative for the quadratic regression coefficient. Values for 
the linear and especially the quadratic regression coefficient are small, because they are multiplied with the day of test and squared day of test, respectively, to yield kilograms of daily feed intake. When comparing estimates of phenotypic standard deviations in Table VI, another problem in the analysis with covariable weight at the end of the test included in the model was discovered. Like fixed effects, covariables are expected to reduce the variance of random effects. Therefore, estimates of phenotypic standard deviation of ADG and FCR were expected to be smaller for model 1 than for model 2. This was the case for ADG, but the estimate of phenotypic standard deviation of FCR obtained with model 1 was higher than estimates from both, model 2 and raw data. Instead of reducing variances of random effects, fitting "weight at the end of the test" as a covariable for FCR seemed to introduce additional variance. The reason for this erratic behaviour of the Gibbs sampler was found in fixing residual variances of single measured traits to a very small value and explicitly fitting residuals as individual permanent environmental effects. Apparently, the Gibbs sampling algorithm was not able to react appropriately if too high values were sampled for the solution $\beta_{n}$ of the covariable "weight at the end of the test" for FCR. Explicitly fitted individual permanent environmental effects must have incorporated the changes of residuals caused by the sample for $\beta_{n}$. Since this had no influence on the fixed residual variance used to set up the mixed model equations, the value for $\beta_{n}$ was not forced to be reduced in the next round of Gibbs sampling. For model 2 without the covariable "weight at the end of the test" for ADG and FCR, no such erratic effects occurred. Cross-classified fixed and random effects seem to be less affected by the missing feedback from the fixed residual variance, since the impact of a change in the solution for one effect on the resulting "phenotypic fit" is much smaller than for covariables. However, fixing the residual variance of single measured traits to a very small value had an impact on the mixing of the Gibbs chain for this model too. In the following, only results from model 2 (without the covariable "weight at the end of the test" for ADG and FCR) will be reported, since the estimates of heritabilities and correlations were influenced by the erratic behaviour of the Gibbs sampler with model 1.

\subsection{Heritabilities and correlations}

\subsubsection{Feed intake curve parameters}

The estimate of 0.32 for the heritability of the intercept regression coefficient of daily feed intake (Tab. VII) is higher than what we found in an earlier study [11], and is identical with the estimate found by Eissen [2] in a two step approach. Heritabilities for linear and quadratic regression coefficients are in the same range as those reported earlier. Phenotypic correlations are very similar to the ones found earlier, but genetic correlations are different 
Table VII. Estimates of posterior means of heritabilities (bold), genetic (above diagonal) and phenotypic (below diagonal) correlations of the intercept, linear and quadratic regression coefficients for daily feed intake, and for single measured performance traits the average daily gain $(A D G)$, feed conversion ratio $(F C R)$, carcass lean content $(C L C)$ and meat quality index $(M Q I)$.

\begin{tabular}{lrrrrrrr}
\hline Trait & Intercept & Linear & Quadratic & $A D G$ & $F C R$ & $C L C$ & $M Q I$ \\
\hline Intercept & $\mathbf{0 . 3 2}$ & -0.02 & 0.83 & 0.82 & 0.50 & -0.33 & -0.04 \\
Linear & -0.40 & $\mathbf{0 . 0 6}$ & -0.35 & 0.38 & 0.48 & -0.55 & 0.57 \\
Quadratic & 0.28 & -0.91 & $\mathbf{0 . 0 3}$ & 0.63 & 0.16 & 0.13 & -0.24 \\
$A D G$ & 0.30 & 0.29 & -0.08 & $\mathbf{0 . 4 5}$ & 0.33 & -0.28 & 0.29 \\
$F C R$ & 0.25 & 0.11 & -0.10 & -0.34 & $\mathbf{0 . 2 1}$ & -0.65 & 0.04 \\
$C L C$ & -0.13 & -0.24 & 0.13 & -0.09 & -0.44 & $\mathbf{0 . 7 9}$ & -0.27 \\
$M Q I$ & 0.01 & 0.03 & 0.01 & 0.02 & 0.08 & -0.11 & $\mathbf{0 . 2 5}$ \\
\hline
\end{tabular}

(Tab. VII). The genetic correlation between the intercept and the quadratic regression coefficient is higher than that reported earlier for another set of Large White data [11], while the genetic correlation between linear and quadratic regression coefficients is lower. Genetic correlations among regression coefficients (Tab. VII) indicate that selection for a higher intercept might lead to flatter feed intake curves. But as heritabilities of linear and quadratic regression coefficients are low, indirect selection responses are expected to be small. This confirms that it is easier to change the overall level than the shape of feed intake curves.

\subsubsection{Weekly means of daily feed intake}

Figure 1 shows the course of heritability, additive genetic, permanent environmental and residual variances for weekly means of daily feed intake. Week 14 is not shown, as only three animals had records in this last week of the test (Tab. I). Course of variances is similar to what we found earlier for Large White and French Landrace pigs [11], but with increased additive genetic and reduced permanent environmental variance. Consequently, heritability estimates are also higher (Fig. 1) than in our previous study [11]. Heritability for weekly means of daily feed intake increased from 0.20 in the first week of the testing period to 0.38 in week 10 (Fig. 1), which is in the range of the values reported by other authors $[4-6,14]$. Because of the relatively high variation around feed intake curves, the heritability for weekly means of daily feed intake is lower in the first seven weeks of the testing period (Fig. 1) than the heritability of the intercept regression coefficient (Tab. VII), which should represent very similar information. Selection for higher feed intake in the beginning of the testing period should thus rather be based on the intercept regression coefficient than on weekly means of daily feed intake of early test weeks. 


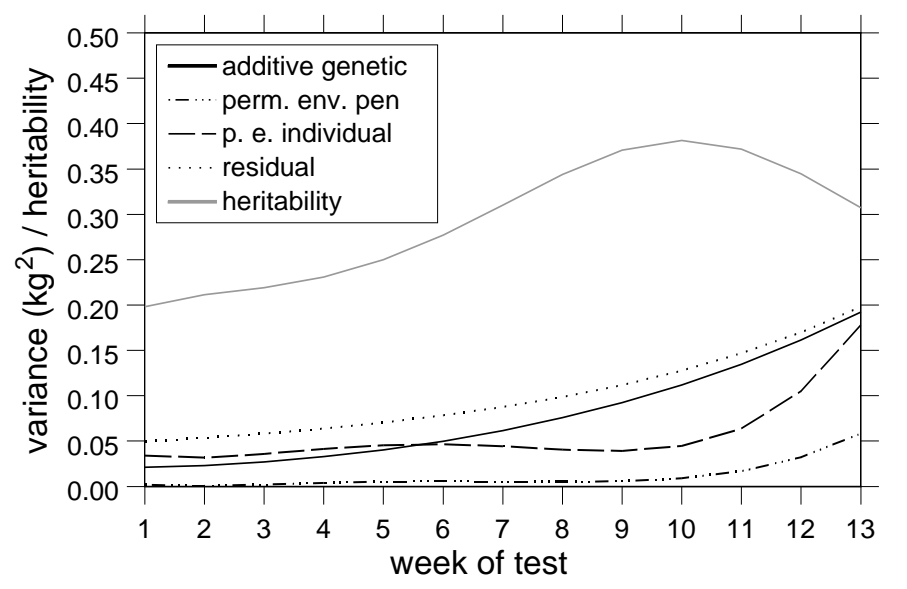

Figure 1. Course of variances and heritability for weekly means of daily feed intake $(\mathrm{kg})$ of Large White growing pigs.

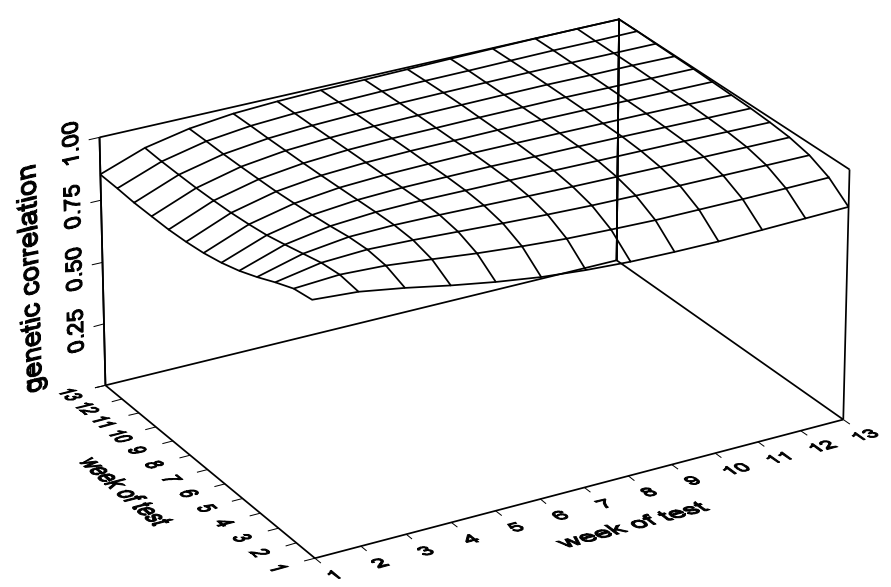

Figure 2. Genetic correlations between weekly means of daily feed intake $(\mathrm{kg})$ of Large White growing pigs.

Genetic correlations between weekly means of daily feed intake (Fig. 2) were very high. The lowest estimates were found for genetic correlations between feed intake in week 1 and feed intake in the second half of the testing period, which were still higher than 0.8 . These values are higher than estimates of Hall et al. [5], who found values between 0.61 and 0.99 using a covariance function model for weekly means of daily feed intake of pigs tested between 45 and $95 \mathrm{~kg}$ live body weight. Estimates of genetic correlations of weekly means of daily feed intake from other studies [6,14], using conventional multiple trait models, are also lower than our estimates from a random regression model. These high 


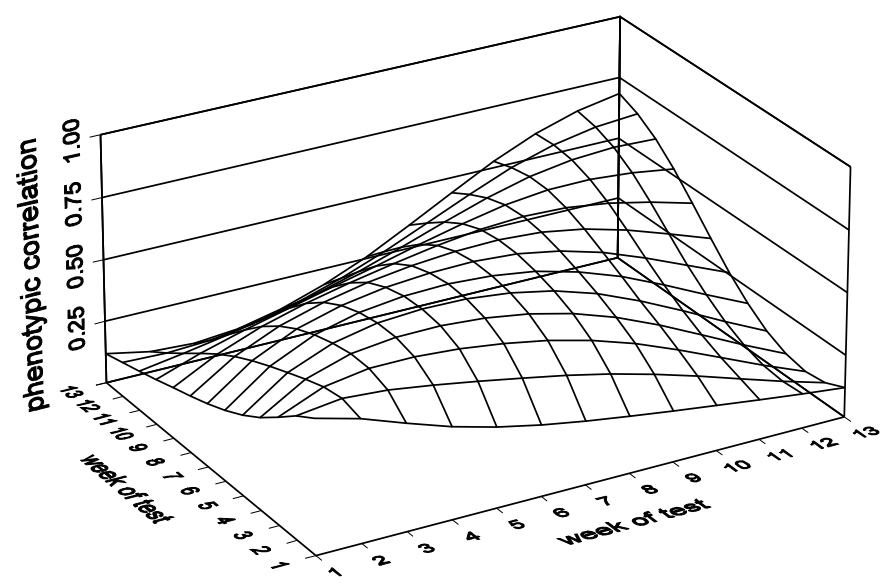

Figure 3. Phenotypic correlations and repeatability (on the diagonal) for weekly means of daily feed intake $(\mathrm{kg})$ of Large White growing pigs.

genetic correlations indicate that selection on daily feed intake at any point during the testing period will result in a similar response over the whole period. Phenotypic correlations between weekly means of daily feed intake (Fig. 3) are substantially lower than genetic correlations. Because of the influence of the residual variance around feed intake curves, (hypothetical) repeated measures of feed intake for the same test week and the same animal need not be the same. The "phenotypic correlations" between records of weekly means of daily feed intake of the same test week, shown on the diagonal in Figure 3, thus represent the repeatability for weekly means of daily feed intake (variance explained by random regression coefficients divided by the total variance). Compared to estimates from regression coefficients alone (based on upper left part of $\left(\mathbf{C}_{\mathbf{G}}+\mathbf{C}_{\mathbf{P}}+\mathbf{C}_{\mathbf{E}}\right)$ in equation (11) only, without residual variances), phenotypic correlations between feed intake records of different test weeks are also reduced due to residual variances. For consecutive test weeks, our estimates are in the same range as those of Hall et al. [5], while they are lower for test weeks that are further apart. Labroue [6] found similar estimates of phenotypic correlations between weekly means of daily feed intake with a multiple trait model, while von Felde et al. [14] estimated higher phenotypic correlations between records in the second part of the testing period.

\subsubsection{Single measured performance traits}

Heritability estimates (Tab. VII) for single measured performance traits are very similar to those found by Labroue et al. [7] for Large White pigs. For model 1 with "weight at the end of the test" included as a covariable for ADG and FCR, lower heritabilities were estimated for ADG (0.33) and FCR (0.09). 
Phenotypic correlations between single measured performance traits (Tab. VII) lie in the range of values found in literature [1,2,4,7,14]. Estimates of genetic correlations (Tab. VII) between CLC and other traits are similar to those found by Labroue et al. [7], while substantial differences (opposite signs) were found for genetic correlations between ADG, FCR and MQI. Eissen [2] estimated genetic correlations between ADG, FCR and CLC, which are similar to our results (Tab. VII). Estimates of Hall et al. [4] (ADG-FCR) and von Felde et al. [14] (ADG-FCR, ADG-CLC) are in the same range as those of Labroue et al. [7]. Genetic correlations between MQI and other traits were closer to those reported by Labroue et al. [7] for French Landrace pigs than for Large Whites.

\subsubsection{Correlations between feed intake parameters and single measured performance traits}

Estimates of phenotypic correlations between regression coefficients for daily feed intake and single measured performance traits varied between -0.24 and 0.30 (Tab. VII). Intercept and linear regression coefficients showed positive phenotypic correlations with ADG and FCR and negative phenotypic correlations with CLC, which are similar to those found by Eissen [2]. Phenotypic correlations of the quadratic regression coefficient with these traits have opposite signs and for MQI phenotypic correlations to regression coefficients for daily feed intake are essentially zero. Genetic correlations of all three regression coefficients with ADG and FCR were positive, while genetic correlations of CLC with intercept and linear regression coefficients were found to be negative. Except for his estimate of 0.25 for the genetic correlation between CLC and the intercept regression coefficient of a linear fit to daily feed intake records, Eissen [2] estimated similar genetic correlations for intercept and linear regression coefficients with these performance traits. Because of this difference (negative correlation with CLC) we are not as optimistic as Eissen [2] about the possible benefits of the intercept regression coefficient for selection. The estimate of the genetic correlation between MQI and the linear regression coefficient was high and positive, while low negative values were found for other regression coefficients. Labroue et al. [7] estimated genetic correlations for average daily feed intake and MQI of 0.00 for Large White pigs and 0.21 for the French Landrace.

Genetic and phenotypic correlations between single measured performance traits and feed intake regression coefficients resulted in almost constant genetic (Fig. 4) and phenotypic (Fig. 5) correlations between performance traits and weekly means of daily feed intake over the whole testing period. Phenotypic and genetic correlations are comparable to the values reported in the literature for phenotypic and genetic correlations between average daily feed intake and other performance traits $[1,2,4,7,14]$. While our phenotypic correlations 


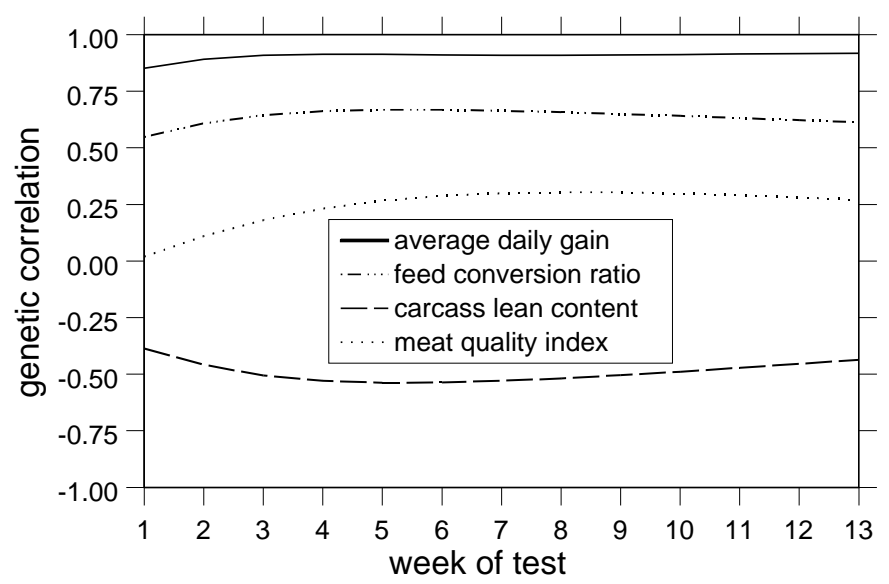

Figure 4. Course of genetic correlations of single measured performance traits with weekly means of daily feed intake $(\mathrm{kg})$ of Large White growing pigs.

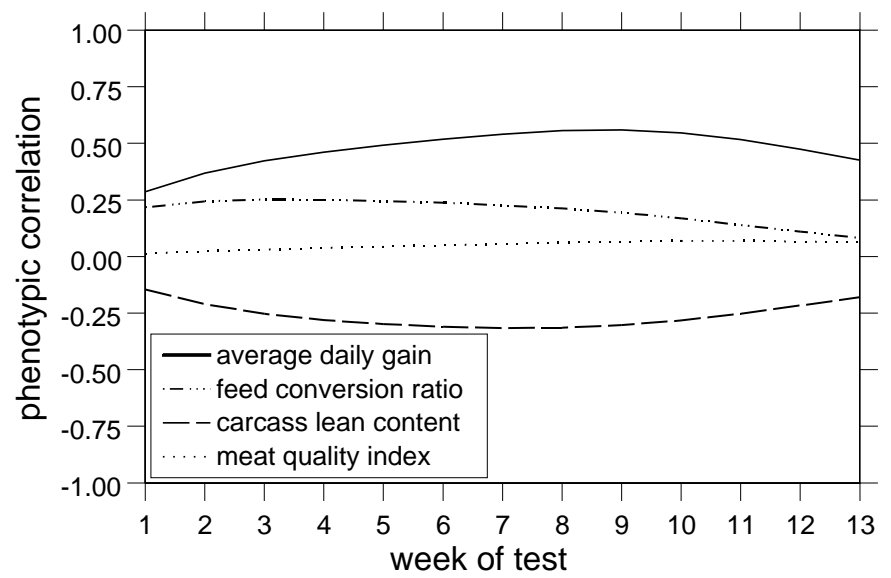

Figure 5. Course of phenotypic correlations of single measured performance traits with weekly means of daily feed intake $(\mathrm{kg})$ of Large White growing pigs.

are situated at the lower end of the range of the values reported, our genetic correlations tend to be slightly higher. Eissen [2] and Hall et al. [4] reported genetic correlations between average daily feed intake and feed conversion ratio similar to our results, while Labroue et al. [7] and von Felde et al. [14] estimated genetic correlations close to zero.

Selection for higher average daily gain and improved meat quality is expected to result in a higher feed intake over the whole testing period, while selection for improved feed conversion (lower FCR) and leaner carcasses is expected to reduce feed intake over the whole testing period. No big differences in the magnitude of these changes were found during the testing period. 
Table VIII. Estimates of standard errors of posterior means (Monte Carlo errors) for heritabilities (bold), genetic (above diagonal) and phenotypic (below diagonal) correlations of the intercept, linear and quadratic regression coefficients for daily feed intake, and for single measured performance traits: average daily gain $(A D G)$, feed conversion ratio $(F C R)$, carcass lean content $(C L C)$ and meat quality index $(M Q I)$.

\begin{tabular}{lccccccc}
\hline Trait & Intercept & Linear & Quadratic & $A D G$ & $F C R$ & $C L C$ & $M Q I$ \\
\hline Intercept & $\mathbf{0 . 0 3 9}$ & 0.047 & 0.036 & 0.047 & 0.102 & 0.052 & 0.030 \\
Linear & 0.001 & $\mathbf{0 . 0 0 2}$ & 0.033 & 0.083 & 0.033 & 0.025 & 0.009 \\
Quadratic & 0.003 & 0.000 & $\mathbf{0 . 0 0 4}$ & 0.105 & 0.084 & 0.040 & 0.052 \\
$A D G$ & 0.008 & 0.006 & 0.007 & $\mathbf{0 . 0 7 3}$ & 0.103 & 0.065 & 0.048 \\
$F C R$ & 0.004 & 0.001 & 0.002 & 0.004 & $\mathbf{0 . 0 1 1}$ & 0.017 & 0.024 \\
$C L C$ & 0.002 & 0.001 & 0.000 & 0.005 & 0.001 & $\mathbf{0 . 0 4 6}$ & 0.025 \\
$M Q I$ & 0.002 & 0.001 & 0.001 & 0.001 & 0.002 & 0.003 & $\mathbf{0 . 0 1 1}$ \\
\hline
\end{tabular}

\subsection{Monte Carlo errors}

Estimates of Monte Carlo errors, i.e. standard errors of posterior means (Tab. VIII) were quite low compared to low estimates of effective sample size (Tab. V). This is due to the high number of samples (2000 000) included in these posterior means. Generally, estimates of standard error of posterior means are lower for phenotypic than for genetic correlations. This is partly due to higher estimates of effective sample size (better mixing of the Gibbs chain), but also because the interval of values visited by the sampler was quite narrow for phenotypic correlations compared to genetic correlations. Despite the fact that estimates of Monte Carlo error (standard deviation of posterior mean) are very low, estimates of heritabilities and correlations should be considered carefully due to high autocorrelations between consecutive samples, which result in low estimates of effective sample size (Tab. V).

\section{CONCLUSIONS}

Explicitly fitting individual permanent environmental effects together with fixing residual variances for single measured traits is a possibility to allow for residual correlations between random regression coefficients and single measured traits in a joint analysis. Estimates of (co)variance components from such models have to be analysed carefully, though, especially if covariables for single measured traits are involved. If no residual correlations between the two types of traits are required, explicitly fitting individual permanent environmental effects for regression coefficients only and allowing for residual correlations between single measured traits should be preferred. 
Heritabilities of random regression coefficients of feed intake curves show that reasonable selection responses can only be expected from the intercept regression coefficient. Changes of slope or inflexion of feed intake curves by direct selection are difficult to achieve. Genetic correlations of feed intake curve parameters with other performance traits are very similar to genetic correlations of average daily feed intake with these traits. Therefore no big advantage is expected from using feed intake regression coefficients instead of average daily feed intake in selection programmes.

\section{ACKNOWLEDGEMENTS}

The authors thank Dr. Karin Meyer, Animal Genetics and Breeding Unit, University of New England, Armidale, Australia for the many valuable comments and discussions that helped to improve this article.

\section{REFERENCES}

[1] De Haer L.C.M., de Vries A.G., Effects of genotype and sex on the feed intake pattern of group housed growing pigs, Livest. Prod. Sci. 36 (1993) 223-232.

[2] Eissen J.J., Breeding for feed intake capacity in pigs. Doctoral thesis, Animal Breeding and Genetics Group, Wageningen University, P.O. Box 338, $6700 \mathrm{AH}$ Wageningen, 2000.

[3] Geyer C.J., Practical Markov chain Monte Carlo, Stat. Sci. 7 (1992) 473-511.

[4] Hall A.D., Hill W.G., Bampton P.R., Webb A.J., Genetic and phenotypic parameter estimates for feeding pattern and performance test traits in pigs, Anim. Sci. 68 (1999) 43-48.

[5] Hall A.D., Bampton P.R., Webb A.J., The effect of stage of growth on the genetic and phenotypic parameter estimates of food intake in pigs using a covariance function model, in: Proceedings of the British Society of Animal Science, March 2000, Annual Meeting Scarborough, 2000, p. 40.

[6] Labroue F., Aspects génétiques du comportement alimentaire chez le porc en croissance. Thèse de Doctorat, Ensar, Rennes, 1996.

[7] Labroue F., Gueblez R., Sellier P., Genetic parameters of feeding behaviour and performance traits in group-housed Large White and French Landrace pigs, Genet. Sel. Evol. 29 (1997) 451-468.

[8] MathSoft, S-Plus, Version 3.4 for UNIX, Supplement, Data Analysis Products Division, MathSoft, Incorporation, Seattle, Washington, 1996.

[9] Meyer K., Hammond K., Mackinnon M.J., Parnell P.F., Estimates of covariances between reproduction and growth in Australian beef cattle, J. Anim. Sci. 69 (1991) 3533-3543.

[10] Raftery A.E., Lewis S., How many iterations in the Gibbs sampler?, in: Bernardo J.M., Berger J.O., Dawid A.P., Smith A.F.M. (Eds.), Bayesian Statistics 4, Oxford University Press, Oxford, 1992, pp. 763-773. 
[11] Schnyder U., Hofer A., Labroue F., Künzi N., Genetic parameters of a random regression model for daily feed intake of performance tested French Landrace and Large White growing pigs, Genet. Sel. Evol. 33 (2000) 635-658.

[12] Sorensen D.A., Andersen S., Gianola D., Korsgaard I., Bayesian inference in Threshold models using Gibbs sampling, Genet. Sel. Evol. 27 (1995) 229-249.

[13] Stahel W.A., Statistische Datenanalyse, Eine Einführung für Naturwissenschaftler, Vieweg, Braunschweig/Wiesbaden, 1995.

[14] von Felde A., Roehe R., Looft H., Kalm E., Genetic association between feed intake and feed intake behaviour at different stages of growth of group-housed boars, Livest. Prod. Sci. 47 (1996) 11-22.

To access this journal online: www.edpsciences.org 